\title{
Short-Term Effects of Flupentixol-Melitracen on Regional Brain Function in Patients with Irritable Bowel Syndrome: An fMRI Study
}

\author{
Jie Li ${ }^{1,2}$, Ping $\mathrm{He}^{3}$, Xingqi Lu ${ }^{2}$, Ying Yang ${ }^{4}$, Yun Guo ${ }^{5}$, Li Li ${ }^{5}$, Yuming Yin ${ }^{6}$, Guoxiong $\mathrm{Li}^{5}$ and Jianping \\ Ding ${ }^{2,4, *}$ \\ ${ }^{1}$ Department of Radiology, The First Affiliated Hospital of Jinan University, Guangzhou, China \\ ${ }^{2}$ Department of Radiology, The Affiliated Hospital of Hangzhou Normal University, Hangzhou, China \\ ${ }^{3}$ Department of Orthodontics, Hangzhou Stomatological Hospital, Hangzhou, China \\ ${ }^{4}$ Medical College, Hangzhou Normal University, Hangzhou, China \\ ${ }^{5}$ Department of Gastroenterology, The Affiliated Hospital of Hangzhou Normal University, Hangzhou, China \\ ${ }^{6}$ MRI Service Committee, Radiology Associate, LLP Corpus Christi, USA \\ "Corresponding author: Department of Radiology, The Affiliated Hospital of Hangzhou Normal University, No. 126, Wenzhou St, Hangzhou, China. Email: 598299041@qq.com
}

Received 2019 March 27; Revised 2019 December 08; Accepted 2020 February 12.

\begin{abstract}
Background: The efficacy of the treatment for irritable bowel syndrome (IBS) with the combination of anxiolytic/antidepressant has been confirmed by clinical studies, but the central mechanism is unclear.

Objectives: To investigate the effects of flupentixol-melitracen, a mixture of tricyclic antidepressant and a classical antipsychotic component, on resting-state brain function in IBS patients by using functional magnetic resonance imaging (fMRI).

Patients and Methods: Thirty-two patients with IBS were randomly divided into an observation group (OG, $\mathrm{n}=16)$ and control group $(\mathrm{CG}, \mathrm{n}=16)$. All patients in both groups were given spasmolytic agent (pinaverium bromide). For the OG group, flupentixolmelitracen was added. FMRI scans were performed in all patients before and four weeks after the therapy. The scores of gastrointestinal symptom rating scale (GSRS), Hamilton depression scale (HAMD-17) and anxiety scale (HAMA-14) were evaluated at the same time. FMRI regional homogeneity (ReHo) parameters were compared between the two groups before and after treatment using a 2 (therapy) $\times 2$ (time) mixed model analysis of variance (ANOVA) statistical method, and were correlated with the changes of GSRS, HAMD and HAMA scores in the two groups.

Results: The fMRI results of the mixed-model analysis of covariance (ANCOVA) and post analyses were as follows. When the therapeutic factor was the main effect, ReHo was increased in the left superior and middle occipital gyrus, and the left parietal gyrus, and decreased in the left thalamus and the lenticular nucleus in both groups after treatment when compared to the unmedicated state. In addition, the OG group showed decreased ReHo in the left superior temporal gyrus, the right lenticular-putamen nucleus, the right inferior frontal gyrus, the right insula, the right gyrus rectus and subcallosal gyrus. The CG group showed increased ReHo in the right angular gyrus. When the interaction between therapy and time was the main effect, the brain region with different ReHo values was in the left precuneus. In the OG group, the change of ReHo in the left precuneus was negatively correlated with the decrease of HAMD and HAMA scores.

Conclusion: Flupentixol-melitracen can effectively improve anxiety, depression and gastrointestinal symptoms in patients with IBS, which may be related to the reversal of abnormal neural activity in the brain regions within the default network and the prefrontal-limbic-temporal emotional circuit in IBS patients.
\end{abstract}

Keywords: Anxiolytic/Antidepressant, Irritable Bowel Syndrome, Functional Magnetic Resonance Imaging, Regional Homogeneity

\section{Background}

Irritable bowel syndrome (IBS) is one of the common gastrointestinal disorders in clinical practice. Its main clinical features are recurrent abdominal pain with changes in bowel habits, but there are no morphological and biochemical abnormalities that can explain it (1). IBS is a chronic visceral pain disorder associated with severe psychological and mental disorders, such as anxiety, de- pression and the occurrence of suicidal ideation, which severely impairs the patient's quality of life (2). Studies showed that anxiety and depression symptoms in IBS patients can affect the processing of visceral stimuli in the central nervous system, leading to further amplification of central pain (3-5). With the recognition of the important role of brain-gut axis in the pathogenesis of IBS, the treatment has shifted from the traditional therapy fo- 
cusing on the terminal target organ (intestinal tract) to the comprehensive treatment including psychobehavioral therapy to adjust the homeostasis of the brain-gut axis. Combined anxiolytic/antidepressant medications can improve the symptoms of mood disorders and effectively relieve abdominal pain in IBS patients, which is better than other conventional treatments, including placebo, spasmodic and antidiarrheal drugs (6-8).

Flupentixol-melitracen is a combination preparation of has both anxiolytic and antidepressant properties and has been proven to be safe and effective in the treatment of IBS $(9,10)$. Flupentixol acts on dopamine receptors in the presynaptic membrane, and increasing the amount of dopamine in the synaptic gap to play an antianxiety and antidepressant role. Small doses of melitracen can inhibit the reuptake of norepinephrine and serotonin in presynaptic membrane, and increase the content of monoamine transmitters in the synaptic space, thereby exerting an antidepressant effect. These two components work synergistically and the side effects cancel each other, so as to effectively and timely improve the neuropsychiatric symptoms, regulate the function of the gastrointestinal autonomic nerves, and reduce visceral hypersensitivity, and finally relieve the physical and mental discomfort.

Rectal stimulation task related functional magnetic resonance imaging (fMRI) study confirmed that tricyclic antidepressant reduces activation of some brain regions during pain which were associated with emotional and cognitive functions (11). Resting state fMRI can be used to detect the activity of brain neurons in resting state, and it is an effective tool to evaluate the central mechanism of therapeutic efficacy (12). The method of regional homogeneity (ReHo) measures the synchronization between time series of a specified voxel and its nearest voxel, thus providing an effective tool to characterize neural activity in resting state at the local level (13). Prior studies of our research group found that IBS patients have ReHo changes in the prefrontal-limbic cortex in resting state (14). To our knowledge, this is the first study to investigate the effects of anxiolytic/antidepressant (flupentixol-melitracen) treatment on regional brain function of IBS patients as indexed by ReHo.

\section{Objectives}

In this study, we hypothesized that combined anxiolytic-antidepressant (flupentixol-melitracen) treatment could reverse abnormal neural activity in the brain regions in IBS patients. Therefore, the purpose of this study was to evaluate the effects of anxiolytic-antidepressant on resting-state brain activity in IBS patients.

\section{Patients and Methods}

\subsection{Study Population}

This trial was supported by the Social Development Project of Zhejiang Public Welfare Technology Research (no.: 2015C33292). The research protocol has been approved by the Ethics Committee of the Affiliated Hospital of Hangzhou Normal University (approval no.: HZNU20160427). Informed written consent was obtained from all participants before their enrollment in this study. Thirty-two native right-handed IBS patients were recruited from the digestive disease clinic of our hospital between June 2016 and May 2018. The diagnosis of IBS for each patient was made by two gastroenterologists based on the Rome III symptom criteria (15). Patients were excluded according to the following criteria: (1) any known food intolerance, malabsorption, history of gastrointestinal surgery, or a history of organic gastrointestinal diseases; (2) antidepressant and prokinetic drugs intake for longer than 2 weeks before enrollment; (3) any current psychiatric disorders (e.g., psychosis, major depressive disorder, or drug and alcohol abuse as defined by diagnostic and statistic manual of mental health disorders, fourth edition text revision [DSM-IV-TR] criteria); (4) history of neurological disorders or head injury; (5) any contraindications to MRI scan. A complete medical history was taken for all patients, and a complete physical examination, blood test, and colonoscopy were performed in all patients.

Using block randomization design, patients were randomly divided into observation group $(\mathrm{OG}, \mathrm{n}=16)$ and control group ( $C G, n=16$ ). All patients in both groups were given spasmolytic agent (pinaverium bromide; $50 \mathrm{mg} /$ day, tid), the OG group was added flupentixol-melitracen (10.5 $\mathrm{mg}$ (day, bid). There were no significant differences in age, gender, or IBS duration $(\mathrm{P}>0.05)$ between the two groups (Table 1).

\subsection{Data Acquisition}

Evaluation of clinical symptoms at baseline and fMRI scans were completed before patients received medication. After 4 weeks of treatment, patients were reassessed and rescanned with the same process.

All patients were assessed by two trained physicians on their Hamilton depression scale (HAMD-17) $(16,17)$, Hamilton anxiety scale (HAMA-14) $(18,19)$, and gastrointestinal symptoms rating scale (GSRS) (20-22). 


\begin{tabular}{lcccc}
\hline Table 1. Demographic Characteristics of Participants & & & & \\
\hline Characteristic & Observation group & Control group & t value & Pvalue \\
\hline Gender (male/female) & $10 / 6$ & $9 / 7$ & $0.79^{\mathrm{b}}$ & \\
Age, $\mathbf{y}$ & $34.38 \pm 7.47$ & $32.06 \pm 7.17$ & 0.893 & 0.379 \\
Duration, mo & $20.38 \pm 11.92$ & $18.06 \pm 9.61$ & 0.604 & 0.550 \\
\hline
\end{tabular}

${ }^{\mathrm{a}}$ Values are expressed as mean $\pm \mathrm{SD}$

${ }^{\mathrm{b}} \chi^{2}$ Pearson chi-square test.

MRI images were acquired by using a whole-body 3.0 T scanner (GE Discovery MR-750, Waukesha, WI) with a 12-channel head coil. The collected images include the resting-state functional blood-oxygen-level-dependent (BOLD) images and high-resolution three-dimensional T1weighted (3D-T1) image. Details of sequences and parameters used for image acquisition are shown in Table 2. Conventional MRI images were reviewed by two experienced radiologists, and no significant structural abnormalities were found in all subjects.

\subsection{Data Analysis}

The fMRI data analyzer was blinded to the treatment protocols and time session of each data. Resting-state fMRI data pre-processing was performed using the Statistical Parametric Mapping (SPM8, http://www.fil.ion.ucl.ac.uk/spm/) and Data Processing Assistant for resting state-fMRI (data processing and analysis for resting state brain imaging [DPABI]) tool (23). Pre-processing: the image data of the first 10 time points of the resting-state scan are removed for slice-timing adjustment. Head-motion correction is eliminate the head motion in the $\mathrm{X}, \mathrm{Y}$, and $\mathrm{Z}$ axis of translation for more than $3 \mathrm{~mm}$ or rotation angle more than $3^{\circ}$ data subjects; spatial standardization was carried out to warped the image into Montreal Neurological Institute (MNI) template $(3 \times 3 \times$ $3 \mathrm{~mm}^{3}$ ). Subsequently, Linear drift of data was removed and $0.01 \sim 0.08 \mathrm{~Hz}$ bandpass filter was used to eliminate physiological noise. By multiple linear regressions, several nuisance variables were removed from the data, including head-motion parameters, global brain signal, and average signal from white matter and ventricles.

After preprocessing, ReHo analysis was performed on the resting-state fMRI data. First, the Kendall's coefficient on the time series of each voxel of the whole brain and its neighboring 26 voxels was calculated, which is the ReHo value of the voxels. Each voxel corresponded to a ReHo value, thus forming a whole-brain ReHo graph. The ReHo value of each voxel in the entire brain was divided by the mean value of all voxel ReHo values in the whole brain and standardized to obtain the average ReHo graph. Then spatial smoothing (full width at half maximum, FWHM $=6$ $\mathrm{mm}$ ) was performed before statistical analysis (13).

\subsection{Statistical Analysis}

Demographic and clinical data were analyzed using SPSS software (Version 18.0, SPSS Inc., Chicago, USA). Differences between group demographic data were analyzed using independent $t$-tests and $\chi^{2}$ tests. Differences in clinical data between groups were analyzed using independent $t$-tests at the pre-treatment and post-treatment. A 2 (therapy) $\times 2$ (time) mixed model analysis of variance (ANOVA) was used to compare pre-treatment and posttreatment differences in the observation group and control group. Significance was set at $\mathrm{P}<0.05$. Statistical analyses of ReHo maps were performed using DPABI. To examine differences in ReHo values among patients with different medications, we performed a voxel wise mixed-model analysis of covariance (ANCOVA) with time (pre-treatment and post-treatment) and therapy (OG and $\mathrm{CG}$ ) as factors. Post analyses were carried out using two-sample $t$-tests. Age, gender, framewise displacement (FD) and IBS duration were included as covariates. The statistical threshold was set at voxel level, $\mathrm{P}<0.01$; mass level, $\mathrm{P}<0.05$, with AlphaSim correction (24).

Finally, partial correlation analysis was performed to examine the association between changes in ReHo following treatment and reduced HAMD, HAMA and GSRS scores, controlling for age, sex, FD and IBS duration. The levels of significance were set at $\mathrm{P}<0.05$ without correction for multiple tests.

\section{Results}

\subsection{Demographic Characteristics and Behavioral Results}

Demographic details and clinical characteristics of all patients were summarized in Table 1 and Table 3. There were no statistically significant differences in GSRS, HAMD and HAMA scale scores between two groups before treatment $(\mathrm{P}>0.05)$ (Table 3), indicating that the two groups 


\begin{tabular}{lcccccc}
\hline \multicolumn{1}{l}{ Table 2. MRI Sequences and Parameters } & & & & \\
\hline & Sequences & TR/TE, $\mathbf{m s}$ & FOV, $\mathbf{m m}$ & Slice thickness, $\mathbf{m m}$ & Matrix $^{\text {FA, }}$ \\
\hline BOLD images & GRE-SS-EPI & $2000 / 30$ & $192 \times 192$ & 4 & $64 \times 64$ & 90 \\
3D-T1 image & SPGR & $8.16 / 3.18$ & $256 \times 256$ & 1 & $256 \times 256$ \\
\hline
\end{tabular}

Abbreviations: BOLD, blood-oxygen-level-dependent; FA, flip angle; FOV, field of view; GRE-SS-EPI, gradient-echo single-shot echo planar imaging; SPGR, spoiled gradientrecalled pulse; 3D-T1, three-dimensional T1-weighted; TR/TE, repetition time/echo time.

had comparable levels of gastrointestinal symptoms, anxiety and depression before treatment. After 4 weeks of treatment, however, analysis of each subtest indicated that the observation group had significantly lower scores in GSRS $(t=-4.122, \mathrm{P}<0.001)$, HAMD $(t=-3.859, \mathrm{P}=0.001)$, and $\operatorname{HAMA}(t=-3.709, \mathrm{P}=0.001)$ compared to the control group. Two-factor mixed design ANOVA for GSRS ( $\mathrm{F}=5.819, \mathrm{P}=$ 0.019), $\operatorname{HAMD}(\mathrm{F}=7.707, \mathrm{P}=0.007)$ and $\operatorname{HAMA}(\mathrm{F}=6.476, \mathrm{P}$ $=0.014$ ) scale scores also showed significant time and therapy interaction effects (Table 3). Compared with the baseline level, the scores of GSRS, HAMD and HAMA scales in the OG group were significantly reduced $(\mathrm{P}<0.001)$, and GSRS score was significantly decreased in CG group after treatment $(\mathrm{P}<0.05)$. HAMD and HAMA score did not show a statistically significant difference in the CG group before and after treatment $(\mathrm{P}>0.05)$.

\subsection{Results of ReHo Comparisons in Mixed-Model ANCOVA and Post Analyses}

\subsubsection{Therapeutic Factor Was the Main Effect}

Following treatment, both groups exhibited significantly increased ReHo in the left superior and middle occipital gyrus, and the left parietal gyrus, whereas decreased ReHo was observed in the left thalamus and the lenticular nucleus. In addition, the OG group showed decreased ReHo in the left superior temporal gyrus, the right lenticular and putamen nucleus, the right inferior frontal gyrus, the right insula, the right gyrus rectus and the right subcallosal gyrus. The CG group showed increased ReHo in the right angular gyrus (AlphaSim corrected, with the level of voxle $\mathrm{P}<0.01$ and the level of mass $\mathrm{P}<0.05$ ) (Figure 1 and Table 4).

\subsubsection{The Interaction Between Therapy and Time Was the Main Effect}

The brain region with statistical difference was left precuneus (Figure 2A) (AlphaSim corrected, with the level of voxle $\mathrm{P}<0.01$ and the level of mass $\mathrm{P}<0.05)$. Compared with baseline level, ReHo in the left precuneus in the OG group was increased, while that in CG group was decreased after treatment (Figure 2B). The changes of ReHo in the left precuneus were negatively correlated with reduction in the HAMD and HAMA scores in the OG group $(\mathrm{r}=-0.712$, $\mathrm{P}=0.009 ; \mathrm{r}=-0.646, \mathrm{P}=0.023$ ) (Figure $2 \mathrm{C}$ and Figure $2 \mathrm{D}$ ).

\section{Discussion}

In the present study, we found that the combination of flupentixol and melitracen was more effective in improving anxiety, depression and gastrointestinal symptoms in IBS patients compared with basic treatment, further confirming that psychotropic drugs can not only control their own anxiety and depressive symptoms, but also contribute to significantly improving gastrointestinal symptoms in IBS patients. We found that both combined with anxiolytic/antidepressant group and only gastrointestinal spasmolytic drug group reduced resting-state brain activity in regions related to "pain matrix", i.e., thalamus and lenticular nucleus, and increased in the posterior parietooccipital cortex. In addition, the OG group resulted in more intensive and extensive decreases in brain activity in the cortices (prefrontal-temporal regions) and the limbic system, while the CG group resulted in greater increases in brain activity in the right angular gyrus. Significantly, we found that anxiolytic/antidepressant treatment was associated with increased ReHo in the left precuneus, and changes in this area were negatively correlated with the reduction in the HAMD and HAMA score.

The left precuneus is the "hub" of the default mode network (DMN) (25). DMN maintains the memory, attention and cognitive functions of the brain in resting state, which is an important node of functional connection of various brain regions (26). The precuneus and surrounding posteromedial areas are the highest metabolic brain regions in resting state, which maintain the self-consciousness function in the internal thinking process related to "selfreference" during rest state. The dysfunction of this region may lead to physiological lesions (25). Studies have reported that IBS patients showed high activation in the precuneus and surrounding areas under rectal stimulation, and decreased blood perfusion in this region and its functional connection with multiple brain regions under resting state, which were partially related to the depression score of patients. (27-30). Therapeutic studies 


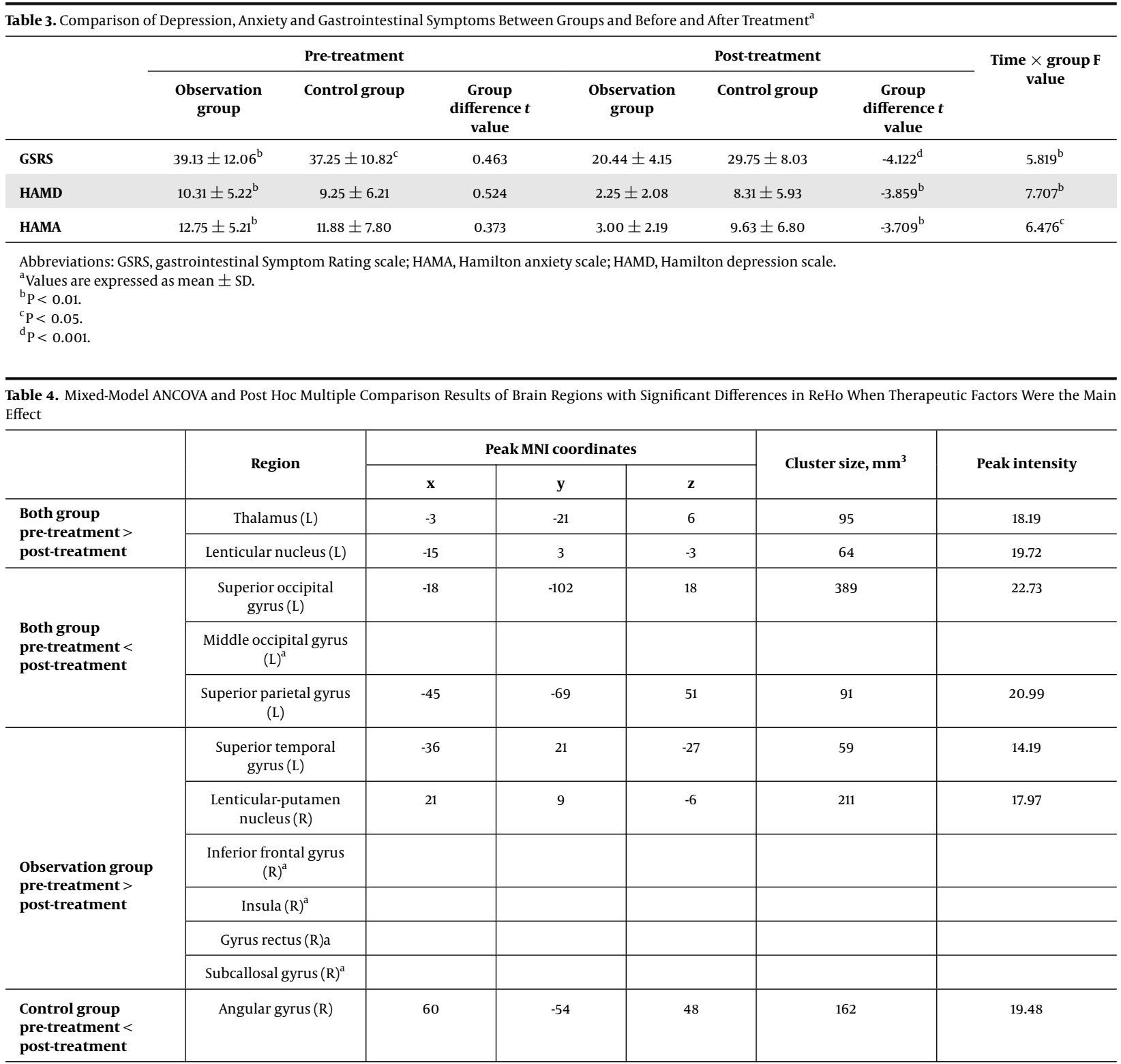

Abbreviations: L, left; MNI, Montreal Neurological Institute; ReHo, Regional homogeneity; R, right.

${ }^{\mathrm{a}} \mathrm{Secondary}$ peaks are in italic.

have shown that abnormal activity and recovery of DMN, especially in the left precuneus, may be a state indicator for predicting anxiety and depression (31-33). When the interaction between therapy and time was the main effect, the region of different brain activity was the left precuneus. It should be noted that spontaneous activity in the precuneus was increased after combined treatment with flupentixol-melitracen, and the regional changes of ReHo values were negatively correlated with the changes of HAMA and HAMD scores, indicating that the increase of regional function correlated with the alleviation of mental symptoms. These results suggested that the precuneus would play a role in the pathophysiology for depression and anxiety; while, flupentixol-melitracen could modulate complex interactions of dopamine and serotonin $(9,10)$ that could also cause upregulation of spontaneous activity in the precuneus. Thus, we speculate that increased spontaneous activity in the precuneus may be a neuromechanism of selective serotonin reuptake inhibitors.

Followed combined anxiolytic/antidepressant treat- 


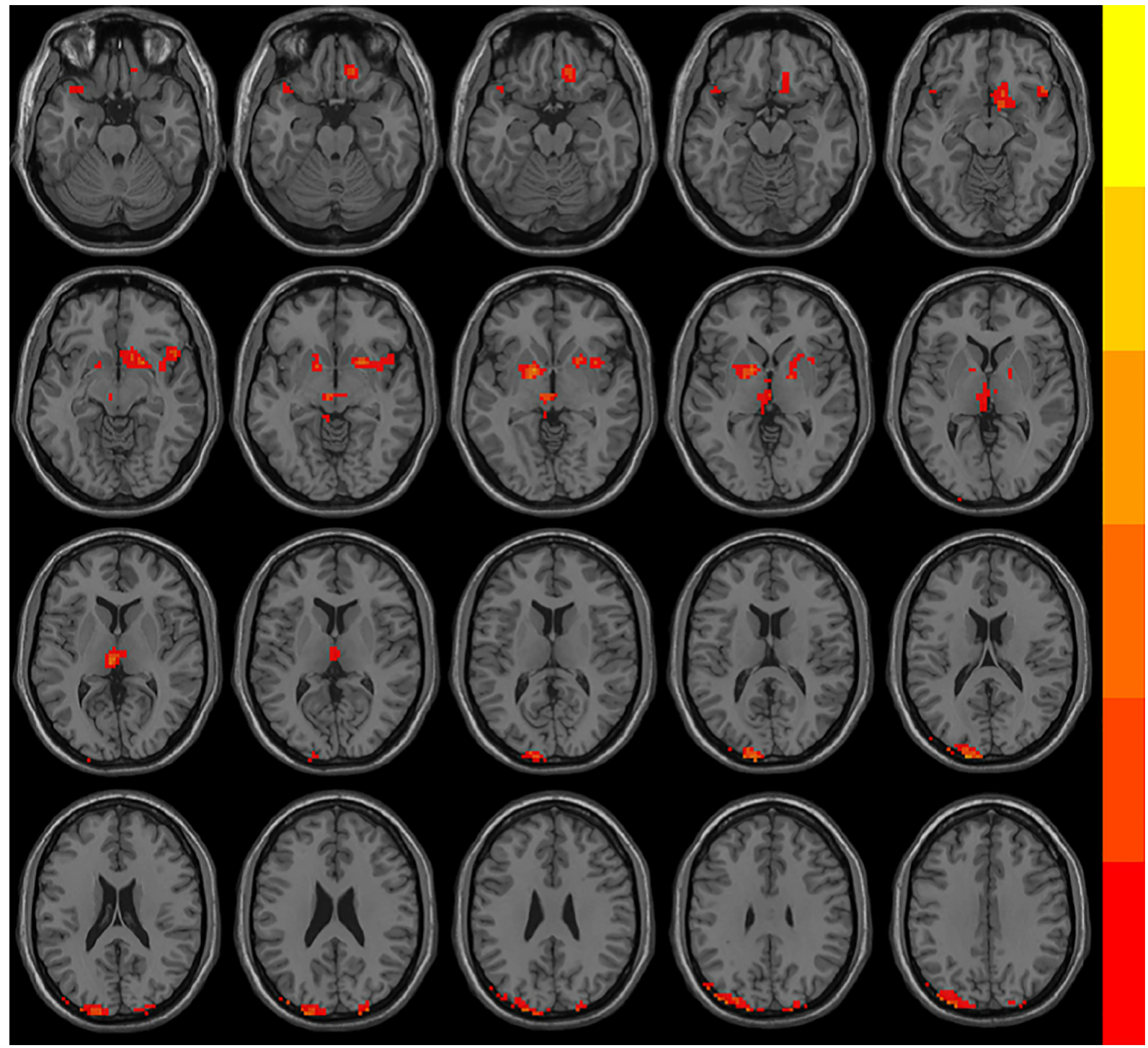

Figure 1. Brain regions with significantly different regional homogeneity (ReHo) values among groups when therapeutic factor was the main effect in the mixed-model analysis of covariance (ANCOVA) analysis $(\mathrm{P}<0.05$, Alphasim corrected). The left side of the images corresponds to the left side of the brain.

ment, IBS patients showed decreased ReHo in the left superior temporal gyrus, the right lenticular-putamen nucleus, the right inferior frontal gyrus, the right insula, the right gyrus rectus and the right subcallosal gyrus. A large number of fMRI studies have shown that abnormal prefrontallimbic emotional circuits, including the prefrontal cortex, anterior cingulate cortex, hippocampus, basal ganglia, amygdala and insula, are potential neuropathological mechanisms for anxiety and depression (34). The major role of antidepressants is to reverse the negative bias in the information processing of the loop, so the neural activity of the loop can predict the efficacy of antide- pressants (35-37). Some studies have found that the degree of atrophy in temporal lobe may be a predictor of the course of depression $(38,39)$, and the high activation of the superior temporal gyrus may be the neural basis for the deficits in cost-benefit decision-making in patients with depression disorder (40). In this study, changes in brain activity in the prefrontal-limbic system and temporal lobe were observed only in the OG group, suggesting that anxiolytic/antidepressant could significantly improve the mood disorders by regulating the emotional processing circuits, further promoting the recovery of gastrointestinal symptoms. The insula in the emotional circuit is 
A

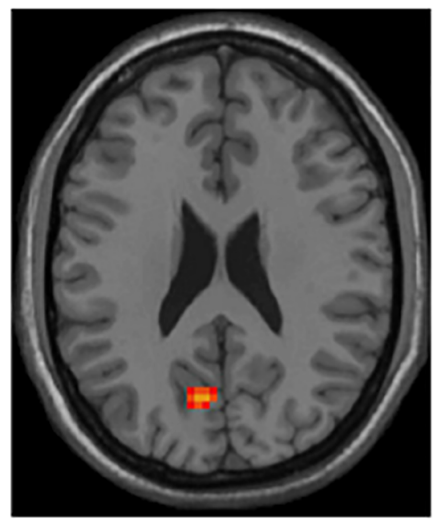

C

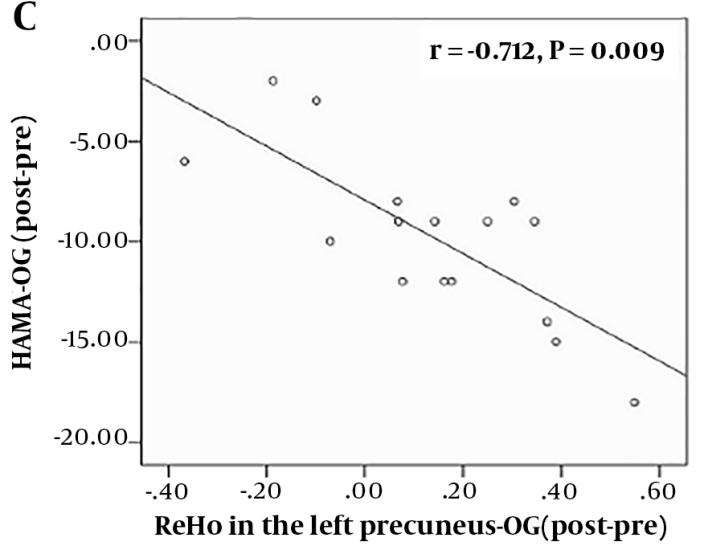

B
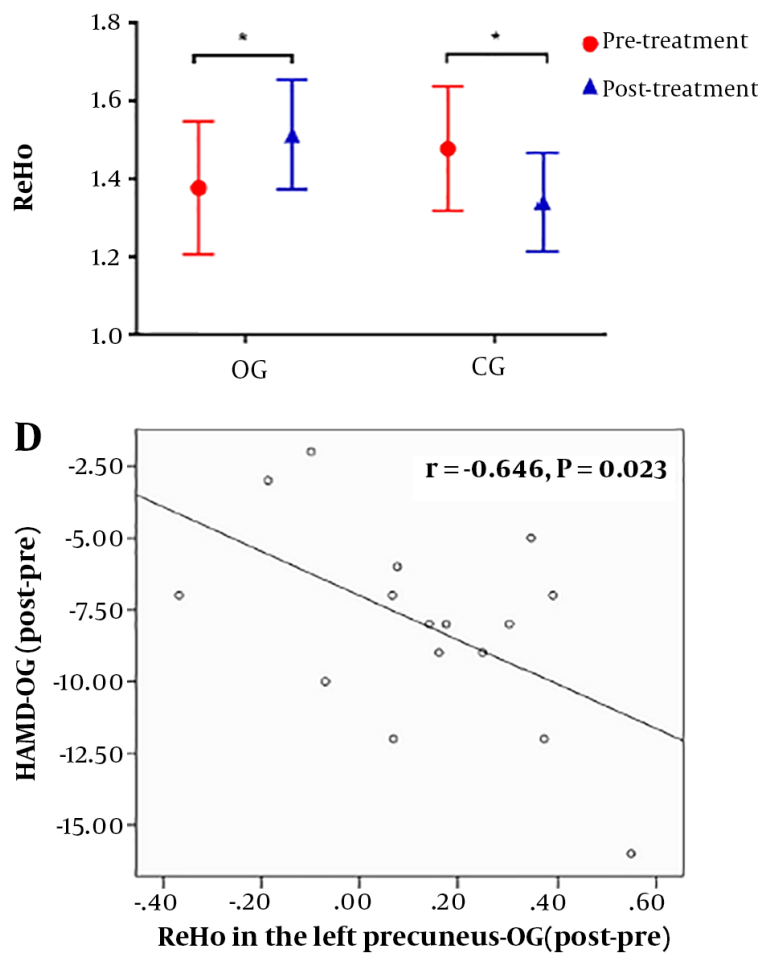

Figure 2. Brain regions with significantly different ReHo when the interaction between treatment and group was the main effect in the mixed-model ANCOVA analysis. A, The region of different ReHo was the left precuneus (red); B, Followed treatment, ReHo in the left precuneus increased in the observation group (OG) and decreased in the control group (CG); C, D, In the OG group, there was a significant negative correlation between the changes of ReHo in the left precuneus and the reduction in the Hamilton depression rating scale (HAMD); and Hamilton anxiety rating scale (HAMA) scores.

not only related to mood change, but also closely related to the production and regulation of pain (41). We found that gastrointestinal symptoms in the CG group were partially relieved after basic treatment, but no significant changes in insular activity were observed. This result may be due to the symptoms of anxiety and depression that have not been significantly alleviated, leading to further amplification of central pain sensation.

When the therapeutic factor was the main effect, the values of ReHo in both groups after treatment were decreased in the left thalamus and the lenticular nucleus, and increased in the superior and middle occipital gyrus, and the left parietal gyrus compared to the unmedicated state. The thalamus is a key component of the steady-state afferent network and plays a crucial role in central processing of somatic and visceral pain. The most distinctive feature of the thalamus is its interconnection with the cerebral cortex, which can transmit pain information to the cerebral cortex (42). Task-state and resting-state fMRI study found that IBS patients had high thalamic activation and increased regional synchronization, which were positively correlated with the severity of gastrointestinal symptoms (43-47). The basal ganglia is involved in the integration of information with the cortex, thalamus and three specific pain processing regions (sensory, emotional/cognitive and endogenous/regulatory), which is an important part of the "pain matrix" $(48,49)$. Lenticular nucleus, an important part of the basal ganglia, serves a significant role in regulating pain (50). Chronic visceral pain can lead to a decrease in the volume of the left putamen (51). The occipital lobe has traditionally been associated with visual information processing. Although no visual impairment associated with chronic pain has been reported, some rodent studies have found that the occipital cortex reflects analgesic effects $(52,53)$. In addition, several human neuroimaging studies have reported changes in occipital lobe activ- 
ity in patients with chronic pain (54-57). The parietal cortex integrates the signals transmitted by various sensory information and is related to sensory perception processing, self-consciousness and memory extraction. Stankewitz et al. (58) found persistent activation of the posterior parietal cortex (including the superior gyrus and subparietal lobules) in the perception of pain spatial location information. The results of our previous study also found ReHo changes in the parietal and occipital cortex in IBS patients (14). Based on the above studies, the findings of our study further indicate that abnormal neuronal activity in the pain network-related brain regions and the posterior parieto-occipital cortex in IBS patients could be recovered after improvement of gastrointestinal symptoms.

In conclusion, anxiolytic/antidepressant could effectively improve anxiety, depression and gastrointestinal symptoms in IBS patients, which may be related to the reversal of abnormal neural activity in the brain regions within the default network and the prefrontal-limbictemporal emotional circuit in IBS patients. In addition, abnormal neuronal activity in the pain network-related brain regions and the posterior parieto-occipital cortex in IBS patients can be recovered after improvement of gastrointestinal symptoms.

This study also had limitations. Frist, the small sample size of this study may have affected the credibility and universality of the research results. Second, long-term follow-up studies are needed to determine whether further changes in clinical symptoms and brain activity occur at later stages. Third, ReHo focuses on the functional areas of the entire brain, but it may be ignoring the functional characteristics of specific brain regions. Last but not the least, the results of this study may be influenced by nontherapeutic factors (for example, the treatment behavior itself leads to differences in the scale and fMRI results). In the future, it is necessary to expand the sample size, improve the consistency of the sample size, add the placebo group, and conduct multimodal research to verify each other.

\section{Footnotes}

Authors' Contributions: Jie Li and Ping He contributed equally to this work. Study concept and design: Jianping Ding, Jie Li, and Ping He. Analysis and interpretation of data: Jie Li, Ping He, Xingqi Lu, and Yun Guo. Drafting of the manuscript: Jie Li and Ping He. Critical revision of the manuscript for important intellectual content: Jianping Ding, Yuming Yin, and Guoxiong Li. Statistical analysis: Jie Li, Ying Yang and Li Li.
Clinical Trial Registration Code: Clinical trial registration code was 2015C33292.

Conflict of Interests: The authors declare that there is no potential conflict of interests regarding the publication of this paper.

Ethical Approval: Approval number was HZNU20160427. Funding/Support: This study was supported by the Social Development project of Zhejiang Public Welfare Technology Research (contract grant numbers: 2015C33292). Hangzhou Medical Science and Technology Program (contract grant numbers: 20190644; 20190233).

Informed Consent: Informed written consent was obtained from all participants before their enrollment in this study.

\section{References}

1. Mearin F, Lacy BE, Chang L, Chey WD, Lembo AJ, Simren M, et al. Bowel disorders. Gastroenterology. 2016;13(9):501-2. doi: 10.1053/j.gastro.2016.02.031. [PubMed: 27144627].

2. Stasi C, Bellini M, Bassotti G, Blandizzi C, Milani S. Serotonin receptors and their role in the pathophysiology and therapy of irritable bowel syndrome. Tech Coloproctol. 2014;18(7):613-21. doi: 10.1007/s10151-0131106-8. [PubMed: 24425100].

3. Garland EL, Gaylord SA, Palsson O, Faurot K, Douglas Mann J, Whitehead WE. Therapeutic mechanisms of a mindfulness-based treatment for IBS: Effects on visceral sensitivity, catastrophizing, and affective processing of pain sensations. J Behav Med.2012;35(6):591-602. doi: 10.1007/s10865-011-9391-z. [PubMed: 22161025]. [PubMed Central: PMC3883954].

4. Wang D, Zhang X, Zhang X, Huang Z, Song Y. Magnetic resonance imaging analysis of brain function in patients with irritable bowel syndrome. BMC Gastroenterol. 2017;17(1):148. doi: 10.1186/s12876-0170673-y. [PubMed: 29216847]. [PubMed Central: PMC5721622].

5. Elsenbruch S, Rosenberger C, Enck P, Forsting M, Schedlowski M, Gizewski ER. Affective disturbances modulate the neural processing of visceral pain stimuli in irritable bowel syndrome: An fMRI study. Gut. 2010;59(4):489-95. doi:10.1136/gut.2008.175000. [PubMed: 19651629].

6. Xiong N, Duan Y, Wei J, Mewes R, Leonhart R. Antidepressants vs. Placebo for the treatment of functional gastrointestinal disorders in adults: A systematic review and meta-analysis. Front Psychiatry. 2018;9:659. doi: 10.3389/fpsyt.2018.00659. [PubMed: 30564156]. [PubMed Central: PMC6288425].

7. Ruepert L, Quartero AO, de Wit NJ, van der Heijden GJ, Rubin G, Muris JW. Bulking agents, antispasmodics and antidepressants for the treatment of irritable bowel syndrome. Cochrane Database Syst Rev. 2011;(8). CD003460. doi: 10.1002/14651858.CD003460.pub3. [PubMed: 21833945].

8. Filipovic BR, Filipovic BF. Psychiatric comorbidity in the treatment of patients with inflammatory bowel disease. World J Gastroenterol. 2014;20(13):3552-63. doi: 10.3748/wjg.v20.i13.3552. [PubMed: 24707138]. [PubMed Central: PMC3974522].

9. Chen H, Lin LM, Zheng JJ. Treatment of diarrhea-predominant postinfectious irritable bowel syndrome with Deanxit: An analysis of 30 cases. World Chin J Digestol. 2009;17(7):733. doi:10.11569/wcjd.v17.i7.733.

10. Qin L, Qin J, Yang Q, Lv X, Huang L. Efficacy and safety of pinaverium bromide combined with flupentixol-melitracen for 
diarrhea-type irritable bowel syndrome: A systematic review and meta-analysis. Medicine (Baltimore). 2019;98(2). e14064. doi: 10.1097/MD.0000000000014064. [PubMed: 30633208]. [PubMed Central: PMC6336559].

11. Morgan V, Pickens D, Gautam S, Kessler R, Mertz H. Amitriptyline reduces rectal pain related activation of the anterior cingulate cortex in patients with irritable bowel syndrome. Gut. 2005;54(5):601-7. doi: 10.1136/gut.2004.047423. [PubMed: 15831901]. [PubMed Central: PMC1774484].

12. Smitha KA, Akhil Raja K, Arun KM, Rajesh PG, Thomas B, Kapilamoorthy TR, et al. Resting state fMRI: A review on methods in resting state connectivity analysis and resting state networks. Neuroradiol J. 2017;30(4):305-17. doi: 10.1177/1971400917697342. [PubMed: 28353416]. [PubMed Central: PMC5524274].

13. Zang Y, Jiang T, Lu Y, He Y, Tian L. Regional homogeneity approach to fMRI data analysis. Neuroimage. 2004;22(1):394-400. doi: 10.1016/j.neuroimage.2003.12.030. [PubMed:15110032].

14. Li J, Li GX, Guo Y, Lu XQ, Li L, Ding JP. [Regional homogeneity in the patients of irritable bowel syndrome complicated with depression: A resting-state functional magnetic resonance imaging study]. Zhonghua Yi Xue Za Zhi. 2018;98(3):196-201. Chinese. doi: 10.3760/cma.j.issn.0376-2491.2018.03.008. [PubMed: 29374914].

15. Rome F. Guidelines-rome III diagnostic criteria for functional gastrointestinal disorders. J Gastrointestin Liver Dis. 2006;15(3):307-12. [PubMed: 17203570].

16. Ehde DM. Hamilton depression rating scale. USA: Elsevier; 2011. p.1205-7. doi:10.1007/978-0-387-79948-3_1989.

17. Zhao JP, Zheng YP. Reliability and validity of Hamilton depression scale assessed in 329 Chinese depression patients. Chin Mentl Health J.1992;5:214-6.

18. Schneider H, Esbitt S, Gonzalez JS. Hamilton anxiety rating scale. In: Gellman M, Turner J, editors. Encyclopedia of behavioral medicine. New York: Springer; 2013.

19. Wang XD, Wang XLHM. Manual of the rating scale for mental health. Chin Ment Health J.1999:253-5.

20. Svedlund J, Sjodin I, Dotevall G. GSRS-a clinical rating scale for gastrointestinal symptoms in patients with irritable bowel syndrome and peptic ulcer disease. Dig Dis Sci. 1988;33(2):129-34. doi: 10.1007/bf01535722. [PubMed: 3123181].

21. Kulich KR, Madisch A, Pacini F, Pique JM, Regula J, Van Rensburg CJ, et al. Reliability and validity of the Gastrointestinal Symptom Rating scale (GSRS) and quality of life in reflux and dyspepsia (QOLRAD) questionnaire in dyspepsia: A six-country study. Health Qual Life Outcomes. 2008;6:12. doi: 10.1186/1477-7525-6-12. [PubMed: 18237386]. [PubMed Central: PMC2276197].

22. Cao YH, Xie DYS. Research progress of functional gastrointestinal disease assessment scale. J Changchun Univ Chin Med. 2015;13(1):210-3.

23. Yan CG, Wang XD, Zuo XN, Zang YF. DPABI: Data processing and analysis for (resting-state) brain imaging. Neuroinformatics. 2016;14(3):33951. doi: 10.1007/s12021-016-9299-4. [PubMed: 27075850].

24. Poline JB, Worsley KJ, Evans AC, Friston KJ. Combining spatial extent and peak intensity to test for activations in functional imaging. Neuroimage. 1997;5(2):83-96. doi: 10.1006/nimg.1996.0248. [PubMed: 9345540].

25. Cavanna AE, Trimble MR. The precuneus: A review of its functional anatomy and behavioural correlates. Brain. 2006;129(Pt 3):564-83. doi: 10.1093/brain/awl004. [PubMed: 16399806].

26. Raichle ME. The brain's default mode network. Annu Rev Neurosci. 2015;38:433-47. doi: 10.1146/annurev-neuro-071013-014030. [PubMed: 25938726].

27. Kano M, Muratsubaki T, Morishita J, Kono K, Mugikura S, Takase K, et al. Influence of uncertain anticipation on brain responses to aversive rectal distension in patients with irritable bowel syndrome. Psychosom Med. 2017;79(9):988-99. doi: 10.1097/PSY.0000000000000484.
[PubMed: 28498276].

28. Hong JY, Naliboff B, Labus JS, Gupta A, Kilpatrick LA, Ashe-McNalley $\mathrm{C}$, et al. Altered brain responses in subjects with irritable bowel syndrome during cued and uncued pain expectation. Neurogastroenterol Motil. 2016;28(1):127-38. doi: 10.1111/nmo.12710. [PubMed: 26526698]. [PubMed Central: PMC4943658].

29. Jia Z, Peng W, Chen Z, Sun H, Zhang H, Kuang W, et al. Magnetization transfer imaging of treatment-resistant depression. $R a$ diology. 2017;284(2):521-9. doi: 10.1148/radiol.2017160820. [PubMed: 28318404].

30. Qi R, Ke J, Schoepf UJ, Varga-Szemes A, Milliken CM, Liu C, et al. Topological reorganization of the default mode network in irritable bowel syndrome.Mol Neurobiol.2016;53(10):6585-93. doi: 10.1007/s12035-0159558-7. [PubMed: 26635086].

31. Quah-Smith I, Suo C, Williams MA, Sachdev PS. The antidepressant effect of laser acupuncture: A comparison of the resting brain's default mode network in healthy and depressed subjects during functional magnetic resonance imaging. Med Acupunct. 2013;25(2):12433. doi: 10.1089/acu.2012.0901. [PubMed: 24761169]. [PubMed Central: PMC3696920].

32. Spies M, Kraus C, Geissberger N, Auer B, Klobl M, Tik M, et al. Default mode network deactivation during emotion processing predicts early antidepressant response. Transl. Psychiatry. 2017;7(1). e1008. doi: 10.1038/tp.2016.265. [PubMed: 28117844].

33. Amen DG, Taylor DV, Meysami S, Raji CA. Deficits in regional cerebral blood flow on brain spect predict treatment resistant depression. $J$ Alzheimers Dis. 2018;63(2):529-38. doi: 10.3233/JAD-170855. [PubMed: 29578481].

34. Disner SG, Beevers CG, Haigh EAP, Beck AT. Neural mechanisms of the cognitive model of depression. Nat Rev Neurosci. 2011;12(8):467. doi: 10.1038/nrn3027. [PubMed: 21731066].

35. Kupfer DJ, Frank E, Phillips ML. Major depressive disorder: New clinical, neurobiological, and treatment perspectives. Lancet. 2012;379(9820):1045-55. doi:10.1016/S0140-6736(11)60602-8.

36. Phillips ML, Chase HW, Sheline YA, Almeida JRC, Deckersbach T, Trivedi $\mathrm{MH}$, et al. Identifying predictors, moderators, and mediators of antidepressant response in major depressive disorder: Neuroimaging approaches. Am J Psychiatry. 2015;172(2):124-38. doi: 10.1176/appi.ajp.2014.14010076. [PubMed: 25640931]. [PubMed Central: PMC4464814].

37. Fonseka TM, Macqueen GM, Kennedy SH. Neuroimaging biomarkers as predictors of treatment outcome in major depressive disorder. J Clin Neurophysiol. 2017;233:21-35. doi: 10.1016/j.jad.2017.10.049. [PubMed: 29150145].

38. Cano M, Martinez-Zalacain I, Bernabeu-Sanz A, Contreras-Rodriguez O, Hernandez-Ribas R, Via E, et al. Brain volumetric and metabolic correlates of electroconvulsive therapy for treatment-resistant depression: A longitudinal neuroimaging study. Transl Psychiatry. 2017;7(2). e1023. doi: 10.1038/tp.2016.267. [PubMed: 28170003]. [PubMed Central: PMC5438019].

39. Bond DJ, Su W, Honer WG, Dhanoa T, Batres YT, Lee SS, et al. Weight gain as a predictor of frontal and temporal lobe volume loss in bipolar disorder: A prospective MRI study. Bipolar Disord. 2019;21(1):50-60. doi: 10.1111/bdi.12722. [PubMed: 30422375].

40. Yang XH, Huang J, Lan Y, Zhu CY, Liu XQ, Wang YF, et al. Diminished caudate and superior temporal gyrus responses to effort-based decision making in patients with first-episode major depressive disorder. Prog Neuropsychopharmacol Biol Psychiatry. 2016;64:52-9. doi: 10.1016/j.pnpbp.2015.07.006. [PubMed: 26192817].

41. Uddin LQ, Nomi JS, Hebert-Seropian B, Ghaziri J, Boucher O. Structure and function of the human insula.J Clin Neurophysiol. 2017;34(4):3006. doi: 10.1097/WNP.0000000000000377. [PubMed: 28644199]. [PubMed Central: PMC6032992]. 
42. Yen CT, Lu PL. Thalamus and pain. Acta Anaesthesiol Taiwan. 2013;51(2):73-80. doi: 10.1016/j.aat.2013.06.011. [PubMed: 23968658].

43. Mertz H, Morgan V, Tanner G, Pickens D, Price R, Shyr Y, et al. Regional cerebral activation in irritable bowel syndrome and control subjects with painful and nonpainful rectal distention. Gastroenterology. 2000;118(5):842-8. doi: 10.1016/s0016-5085(00)70170-3. [PubMed: 10784583].

44. Ringel Y, Drossman DA, Turkington TG, Bradshaw B, Hawk TC, Bangdiwala $S$, et al. Regional brain activation in response to rectal distension in patients with irritable bowel syndrome and the effect of a history of abuse. Dig Dis Sci. 2003;48(9):1774-81. doi:10.1023/a:1025455330704. [PubMed:14561000].

45. Kwan CL, Diamant NE, Pope G, Mikula K, Mikulis DJ, Davis KD. Abnormal forebrain activity in functional bowel disorder patients with chronic pain. Neurology. 2005;65(8):1268-77. doi: 10.1212/01.wnl.0000180971.95473.cc. [PubMed: 16247056].

46. Verne GN, Himes NC, Robinson ME, Gopinath KS, Briggs RW, Crosson $\mathrm{B}$, et al. Central representation of visceral and cutaneous hypersensitivity in the irritable bowel syndrome. Pain. 2003;103(1-2):99-110. doi: 10.1016/s0304-3959(02)00416-5. [PubMed: 12749964].

47. Ke J, Qi R, Liu C, Xu Q, Wang F, Zhang L, et al. Abnormal regional homogeneity in patients with irritable bowel syndrome: A resting-state functional MRI study. Neurogastroenterol Motil. 2015;27(12):1796-803. doi: 10.1111/nmo.12692. [PubMed: 26403620].

48. Price DD. Psychological and neural mechanisms of the affective dimension of pain. Science. 2000;288(5472):1769-72. doi: 10.1126/science.288.5472.1769. [PubMed: 10846154].

49. Legrain $\mathrm{V}$, Iannetti GD, Plaghki L, Mouraux A. The pain matrix reloaded: A salience detection system for the body. Prog Neurobiol. 2011;93(1):111-24. doi: 10.1016/j.pneurobio.2010.10.005. [PubMed: 21040755].

50. Loher TJ, Hasdemir MG, Burgunder JM, Krauss JK. Long-term followup study of chronic globus pallidus internus stimulation for posttraumatic hemidystonia. J Neurosurg. 2000;92(3):457-60. doi: 10.3171/jns.2000.92.3.0457. [PubMed: 10701534].
51. Labus JS, Dinov ID, Jiang Z, Ashe-McNalley C, Zamanyan A, Shi Y, et al. Irritable bowel syndrome in female patients is associated with alterations in structural brain networks. Pain. 2014;155(1):137-49. doi: 10.1016/j.pain.2013.09.020. [PubMed: 24076048]. [PubMed Central: PMC4100785].

52. Reis GM, Dias QM, Silveira JW, Del Vecchio F, Garcia-Cairasco $\mathrm{N}$, Prado WA. Antinociceptive effect of stimulating the occipital or retrosplenial cortex in rats. J Pain. 2010;11(10):1015-26. doi: 10.1016/j.jpain.2010.01.269. [PubMed: 20418174].

53. Rossaneis AC, Reis GM, Prado WA. Stimulation of the occipital or retrosplenial cortex reduces incision pain in rats. Pharmacol Biochem Behav. 2011;100(2):220-7. doi:10.1016/j.pbb.2011.08.024. [PubMed: 21924282].

54. Klug S, Anderer P, Saletu-Zyhlarz G, Freidl M, Saletu B, Prause W, et al. Dysfunctional pain modulation in somatoform pain disorder patients. Eur Arch Psychiatry Clin Neurosci. 2011;261(4):267-75. doi: 10.1007/s00406-010-0148-4. [PubMed: 20924589].

55. Karibe H, Arakawa R, Tateno A, Mizumura S, Okada T, Ishii T, et al. Regional cerebral blood flow in patients with orally localized somatoform pain disorder: A single photon emission computed tomography study. Psychiatry Clin Neurosci. 2010;64(5):476-82. doi: 10.1111/j.1440-1819.2010.02119.x. [PubMed: 20727111].

56. Cauda F, Sacco K, Duca S, Cocito D, D'Agata F, Geminiani GC, et al. Altered resting state in diabetic neuropathic pain. PLoS One. 2009;4(2). e4542. doi: 10.1371/journal.pone.0004542. [PubMed: 19229326]. [PubMed Central: PMC2638013].

57. Li J, Huang X, Sang K, Bodner M, Ma K, Dong XW. Modulation of prefrontal connectivity in postherpetic neuralgia patients with chronic pain: A resting-state functional magnetic resonance-imaging study. $J$ Pain Res. 2018;11:2131-44. doi: 10.2147/JPR.S166571. [PubMed: 30323648]. [PubMed Central: PMC6174681]

58. Stankewitz A, Schulz E, May A. Neuronal correlates of impaired habituation in response to repeated trigemino-nociceptive but not to olfactory input in migraineurs: An fMRI study. Cephalalgia. 2013;33(4):256-65. doi: 10.1177/0333102412470215. [PubMed: 23230239]. 